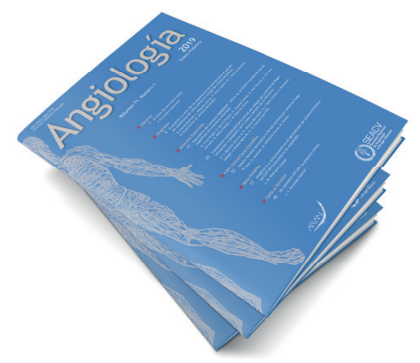

\title{
Original
}

\section{Evaluación in vitro de los dispositivos de energía para el sellado de colaterales de vena safena en cirugía de revascularización}

\section{In vitro model to assess energy devices for the sealing of saphenous vein collaterals in revascularization surgery}

\begin{abstract}
Moisés Falcón Espínola', Lucía Requejo García', Emma Plana Andani², Manuel Ignacio Sánchez Nevárez', Nohelia Rojas Ferrer³, Manuel Miralles Hernández'

${ }^{\top}$ Servicio de Angiología y Cirugía Vascular. Hospital Universitari i Politècnic La Fe. Valencia. Instituto de Investigación Sanitaria La Fe. Hospital Universitari i Politècnic La Fe Valencia. ${ }^{3}$ Servicio de Anatomía Patológica. Hospital Universitari i Politècnic La Fe. Valencia
\end{abstract}

Palabras clave:

Electrocoagulación. Bisturí armónico. Bisturí ultrasónico. Safenectomía.

Key words:

Electrothermal. Harmonic scalpel. Ultrasonic scalpel. Saphenectomy.

\section{Resumen}

Introducción: los dispositivos de sellado por energía permiten la hemostasia de los vasos mediante su coagulación y transección de forma secuencial.

Objetivos: comparar la eficacia in vitro de los principales dispositivos utilizados (electrocoagulación bipolar controlada por temperatura [EB] y bisturí armónico BA]) frente a la ligadura convencional en el sellado de colaterales de vena safena (VS) durante su preparación eventual para la cirugía de bypass.

Material y métodos: estudio experimental in vitro de 25 fragmentos de VS extraída de donante cadáver o remanentes de cirugía de revascularización o amputación. En cada fragmento se realizó un sellado mediante ligadura con seda $3 / 0$ (control) y otro con EB $(n=13)$ o BA $(n=12)$. Cada fragmento se incorporó a un circuito cerrado con flujo pulsátil y se aumentó progresivamente la presión hasta llegar a 300 mmHg (presión suprafisiológica) y, posteriormente, hasta provocar la rotura. Se registró el diámetro de las colaterales, la presión de estallido, el punto de fuga y el estudio histológico.

Resultados: la presión media de estallido fue ligeramente superior para la EB $(788,9 \pm 455 \mathrm{mmHg})$ que para el BA $(602,5 \pm 363,1 \mathrm{mmHg})$, aunque sin diferencias significativas $(p=0,268)$. En un solo caso (BA) se produjo el estallido en la zona de sellado a presiones inferiores a $300 \mathrm{mmHg}$. El punto de fuga para el BA ocurrió en la zona de sellado en todos los casos (12/12), mientras que para la EB se produjo en la zona de sellado en 8 de 13 fragmentos $(p=0,039)$. El estudio histológico no mostró diferencias entre ambos métodos.

Conclusiones: los dispositivos de hemostasia por energía presentan una eficacia y seguridad no inferior al de la ligadura en el sellado de colaterales de VS. Dados su rapidez y fácil manejo, pueden ser útiles en la preparación del injerto venoso durante la cirugía de revascularización. Aunque el EB mostró una mayor resistencia a la rotura al producirse el estallido a presiones suprafisiológicas, este hecho podría no tener relevancia clínica.

\section{Abstract}

Introduction: energy sealing devices achieve hemostasis of the blood vessels through sequential coagulation and transection.

Objectives: to compare the efficacy of the main sealing devices used (Electrothermal Bipolar Tissue Sealing System [EB] and Harmonic Scalpel [HS]) versus conventional vessel ligation of saphenous vein (SV) collaterals.

Recibido: 11/7/2018 • Aceptado: 18/9/2018

Falcón Espínola M, Requejo García L, Plana Andani E, Sánchez Nevárez Ml, Rojas Ferrer N, Miralles Hernández M. Evaluación in vitro de los dispositivos de energía para el sellado de colaterales de vena safena en cirugía de revascularización. Angiología 2019;71(1):2-10.

DOl: http://dx.doi.org/10.20960/angiologia.00016

El siguiente artículo fue presentado como comunicación libre en el LXIV Congreso de la SEACV, celebrado en Madrid del 5 al 8 de junio de 2018.

\section{Correspondencia:}

Moisés Falcón Espínola. Servicio de Angiología y Cirugía Vascular. Hospital Universitari i Politècnic la Fe. Avinguda de Fernando Abril Martorell, 106. 46026 Valencia e-mail: mfalcon6@gmail.com 


\begin{abstract}
Material and methods: experimental in vitro study of 25 fragments of SV extracted from cadaveric donor, from residual fragments obtained during amputation or lower limb revascularization procedures. Two venous collateral seals were made on each fragment, one by conventional ligation with 3/0 silk (control) and the other one by EB $(n=13)$ or HS $(n=12)$. Each venous fragment was then incorporated into a pulsatile flow circuit, and the pressure was progressively increased until $300 \mathrm{mmHg}$ (supraphysiological pressure) was reached, and, later on, until sealing breakage occurred. Collateral vein diameter, burst pressure, and leakage points were recorded. A histological study was also performed for each energy sealing device.
\end{abstract}

Results: the mean burst pressure was slightly higher for $\mathrm{EB}(788,9 \pm 455 \mathrm{mmHg})$ than for $\mathrm{HS}(602,5 \pm 363,1 \mathrm{mmHg})$, but without significant differences $(p=0,268)$. Only in one case (HS) the outbreak occurred in the sealing zone at pressures below $300 \mathrm{mmHg}$. The leakage point for HS was detected in the sealing zone in all cases (12/12). The leakage point for EB occurred in the sealing zone in 8 of 13 fragments $(p=0,039)$. The histological study showed no differences.

Conclusions: Vessel sealing devices are as effective for the sealing of saphenous vein collaterals as conventional ligation. These devices may be useful due to their fast sealing time and easy handling during surgical venous graft preparation for lower limb revascularization. Although the EB showed greater strength, the outbreak occurred at supraphysiological pressure, so this fact may not have clinical relevance.

\section{INTRODUCCIÓN}

Los dispositivos de sellado por energía permiten la hemostasia de los vasos sanguíneos mediante coagulación y transección del vaso de forma secuencial a través de sus distintas propiedades físicas.

Los dos principales sistemas de sellado por energía empleados actualmente son la electrocoagulación bipolar controlada por temperatura (EB) y el bisturí armónico o bisturí de ultrasonidos (BA). El mecanismo de sellado mediante EB está basado en la aplicación de energía entre las dos puntas del dispositivo, lo que produce la desnaturalización del colágeno y elastina de la pared del vaso y de los tejidos circundantes. Las ventajas de este sistema con respecto a otros métodos de electrocoagulación es la capacidad de calcular la impedancia del tejido clampado y ajustar la energía necesaria para conseguir la hemostasia sin llegar al estadio de carbonización tisular. Posteriormente, se realiza el corte gracias a una cuchilla incorporada al sistema. Este dispositivo permite el sellado seguro de vasos de hasta $7 \mathrm{~mm}$ de diámetro $(1,2)$.

El bisturí armónico (BA), o bisturí de ultrasonidos, produce el sellado de los vasos sanguíneos mediante el uso de energía eléctrica para la excitación de materiales piezoeléctricos en la punta de su hoja y la transformación de esa vibración de alta frecuencia en calor, lo que permite la coagulación y el corte del tejido. Este sistema permite el sellado de vasos de hasta $5 \mathrm{~mm}$ de diámetro $(3,4)$.

Aunque existen varios estudios que han demostrado la eficacia y la seguridad de estos sistemas hemostáticos en modelos animales y humanos, se han centrado sobre todo en la cirugía abdominal y torácica endoscópica (5-9). Existe poca experiencia en el ámbito de la cirugía vascular, y aún en menor medida en la cirugía de revascularización, dada la baja implantación de la endoscopia en nuestra especialidad.

En los últimos años han aparecido nuevos abordajes quirúrgicos mínimamente invasivos, como es la safenectomía endoscópica en la cirugía de revascularización coronaria o de miembros inferiores, con resultados prometedores en cuanto a disminución de complicaciones de la herida quirúrgica (10-12). No obstante, su difusión se ha visto limitada por la falta de estudios que avalen su eficacia en el sellado de las colaterales venosas sometidas a presión arterial como método alternativo a su ligadura o clipado directo. De hecho, la mayor parte de cirujanos siguen recurriendo a los métodos clásicos durante la preparación de injertos autólogos o heterólogos antes de la cirugía de bypass.

El objetivo de este estudio consistió en comparar la eficacia in vitro de los principales dispositivos de sellado por energía utilizados (electrocoagulación bipolar controlada por temperatura y bisturí armónico) frente a la ligadura convencional en el sellado de colaterales de vena safena (VS).

\section{MATERIAL Y MÉTODOS}

Estudio experimental en un modelo hemodinámico in vitro para evaluar la seguridad y la eficacia 


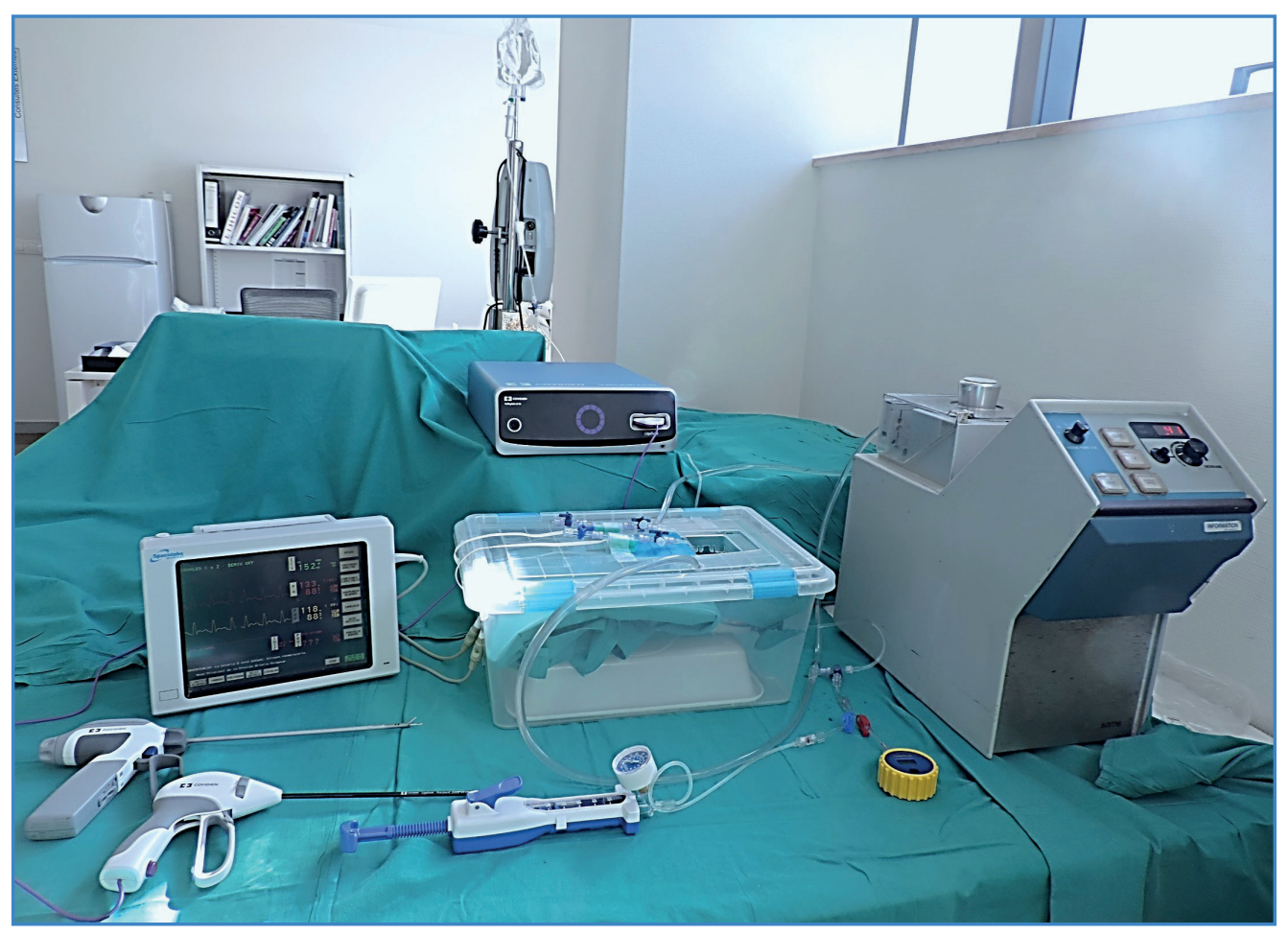

Figura 1. Circuito de flujo pulsátil utilizado para el estudio y el registro de la presión con monitor y manómetro. En la esquina inferior izquierda se visualizan los dos dispositivos de sellado por energía.

de 2 métodos de sellado vascular (EB y BA) en 25 fragmentos de VS interna. A cada fragmento se le aplicó un dispositivo de sellado (intervención) por asignación secuencial consecutiva.

Los fragmentos de VS interna se extrajeron de un cadáver donante $(n=6)$ o de VS remanente de cirugía de revascularización o amputación mayor ( $\mathrm{n}=$ 19), previo consentimiento informado del paciente y de los familiares del donante según los protocolos habituales. Cada fragmento de VS interna de aspecto sano se sumergió en suero fisiológico heparinizado y se congeló a $-15^{\circ} \mathrm{C}$ hasta su uso.

En el momento del estudio, los fragmentos se descongelaron lentamente a temperatura ambiente y se calentaron en baño hasta $37^{\circ} \mathrm{C}$.

Cada fragmento de VS se dividió en fragmentos venosos de menor tamaño con, al menos, dos colaterales. En cada fragmento se realizaron dos sellados: una colateral mediante ligadura con seda (control) y la otra con EB $(n=13)$ o BA $(n=12)$. Para el sellado de las colaterales, se empleó como sistema de EB el dispositivo Ligasure ${ }^{\circledR}$ (Maryland Jaq Sealer/Divider -Covidien, Colorado, Estados Unidos-) y su gene- rador Valleylab ${ }^{\circledR}$ LS10, mientras que para el BA se empleó el dispositivo Sonicision ${ }^{\circledast}$ (Cordless Ultrasonic Dissector -Covidien, Colorado, Estados Unidos-). Como control, se utilizó una hebra de seda de 3/0 (Lorca Marín, España) y ligadura mediante nudo quirúrgico convencional.

Para simular condiciones fisiológicas, se utilizó un circuito cerrado con flujo pulsátil generado por una bomba de rodillo a 0,34 rpm, con una frecuencia de pulso simulado de 80 ppm y una presión controlada del sistema de 130/100 mmHg. Se emplearon tubos de circuito extracorpóreo de 1/4"y una bomba de rodillo (Sarns ${ }^{\circledR}$ Ann Arbor -Michigan, Estados Unidos-) (Fig. 1). Para la interposición del fragmento venoso se empleó un sistema de llave de tres pasos con dos cánulas de oliva y fijación del injerto venoso proximal y distalmente con hebra de seda de 2/0 (Lorca Marín, España). El medio de fluido fue solución salina isotónica.

Se registró la presión intravascular mediante un monitor de presión $(\mathrm{mmHg}$ ) y un manómetro industrial (bar) (GOD0002 -Goodyear ${ }^{\circledR}$, Ohio, Estados Unidos-) para presiones superiores a $300 \mathrm{mmHg}$. 

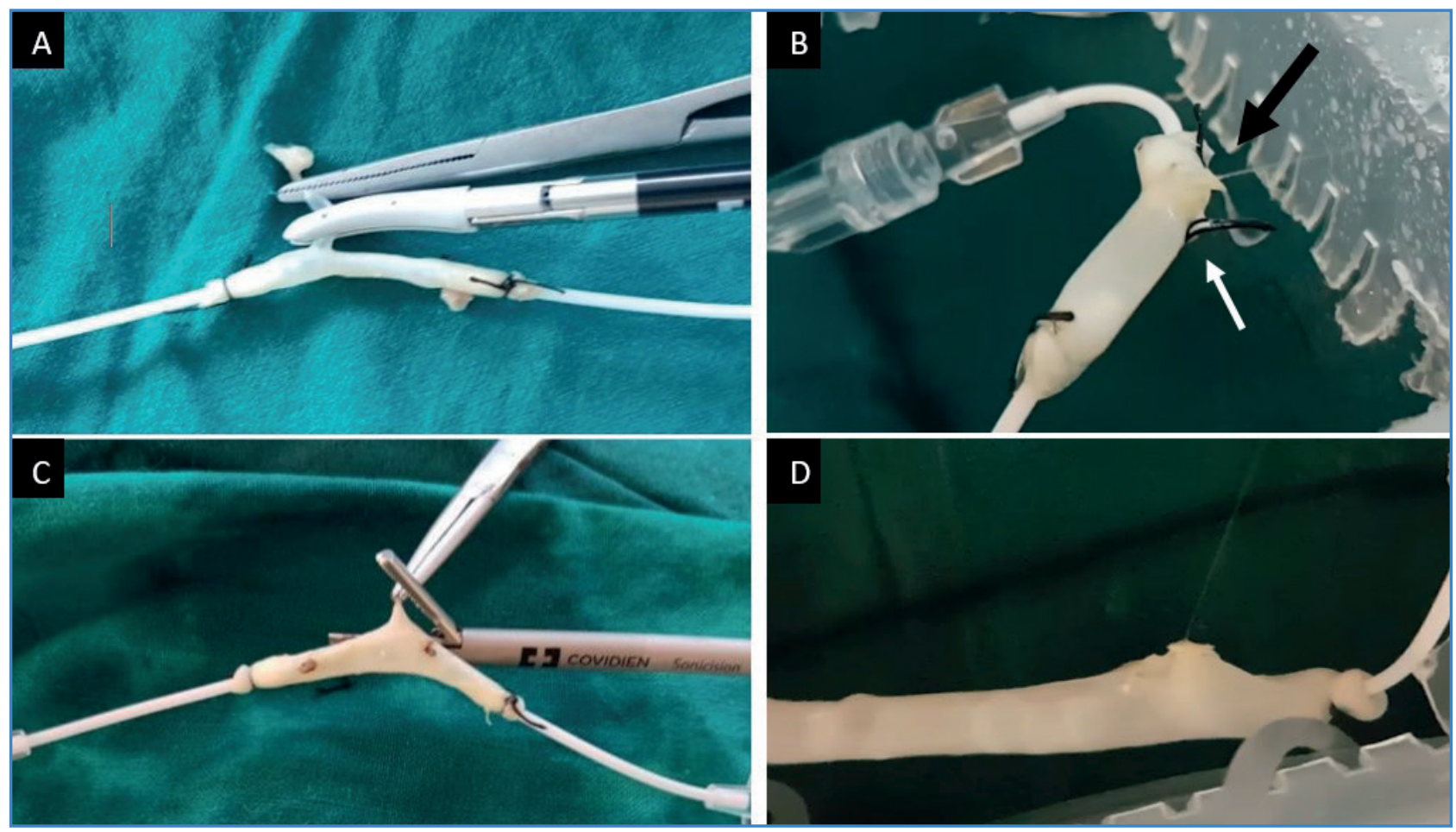

Figura 2. Sellado de colaterales venosas con dispositivos de hemostasia por energía. A. Sellado de colateral venosa mediante electrocoagulación bipolar controlado por temperatura (EB). B. Estallido de colateral venosa sellada por EB (flecha negra) con estanquidad de colateral con ligadura convencional (flecha blanca). C. Sellado con bisturí armónico. D. Estallido del sellado por BA.

Posteriormente, se interpuso cada fragmento en el circuito de flujo pulsátil y se aumentó progresivamente la presión intravascular mediante una jeringa manómetro Encore ${ }^{\circledR}$ (Boston Scientific, Estados Unidos) hasta alcanzar $300 \mathrm{mmHg}$ en primer lugar y, a continuación, hasta provocar la rotura de la zona de sellado o ligadura (Fig. 2).

Como variable principal del estudio se registró la presión de estallido, que se definió como el último registro de presión hasta el momento en el que se produjo la rotura y fuga del suero fisiológico a través del fragmento venoso. Como variables secundarias se registraron el punto de fuga y el diámetro de la colateral venosa.

Asimismo, se realizó el estudio histológico de las zonas de sellado de ambos dispositivos mediante tinciones de hematoxilina-eosina, tricrómico de Masson y elastina.

Para el cálculo estadístico descriptivo y analítico se utilizó el paquete estadístico SPSS ${ }^{\circledR}$ (IBM) en su versión 22.0. Para las comparaciones de los resultados de ambos dispositivos, se emplearon pruebas no paramétricas según criterios de normalidad de Kolmogorov-Smirnov (U Mann-Whitney, F de Fisher).

\section{RESULTADOS}

Los resultados individuales para cada dispositivo y experimento aparecen reflejados en la tabla I.

La presión media de estallido fue ligeramente superior para la EB $(788,9 \pm 455 \mathrm{mmHg})$ que para el $\mathrm{BA}$ $(602,5 \pm 363,1 \mathrm{mmHg})$, aunque sin diferencias significativas ( $p=0,268)$. En solo un caso de BA, la presión de estallido en su zona de sellado fue inferior a $300 \mathrm{mmHg}$. El diámetro medio de las colaterales venosas para la EB fue de 2,4 $\pm 0,7 \mathrm{~mm}$, mientras que para el BA fue de $2,4 \pm 0,6 \mathrm{~mm}(p=0,852)$. Tampoco se encontró una correlación significativa entre el diámetro de la colateral y la presión de estallido $(r=0,093 ; p=0,567)$. El punto de fuga para el BA se produjo en la zona del sellado del dispositivo en 12 de 12 fragmentos, mientras que para la EB el punto de fuga se identificó en dicho punto en 8 de 13 segmentos $(p=0,039)$ (Tabla II). 
Tabla I. Resultados y características individuales de los fragmentos venosos evaluados tras el sellado con diferentes dispositivos de hemostasia por energía

\begin{tabular}{|c|c|c|c|c|}
\hline & Dispositivo & $\begin{array}{l}\text { Diámetro de colateral } \\
\qquad(\mathrm{mm})\end{array}$ & $\begin{array}{l}\text { Presión de estallido } \\
(\mathrm{mmHg})\end{array}$ & Punto de fuga \\
\hline 1 & $E B$ & 2 & 1200 & Ligadura \\
\hline 2 & BA & 1,5 & 488 & Sellado BA \\
\hline 3 & $E B$ & 1,5 & 488 & Sellado EB \\
\hline 4 & BA & 2,5 & 375 & Sellado BA \\
\hline 5 & $E B$ & 1,5 & 600 & Sellado EB \\
\hline 6 & BA & 4 & 750 & Sellado BA \\
\hline 7 & $E B$ & 2,5 & 975 & Ligadura \\
\hline 8 & BA & 2 & 488 & Sellado BA \\
\hline 9 & $E B$ & 2 & 638 & Sellado EB \\
\hline 10 & BA & 2,5 & 338 & Sellado BA \\
\hline 11 & $\mathrm{~EB}$ & 2 & 1088 & Sellado EB \\
\hline 12 & BA & 2,5 & 1315 & Sellado BA \\
\hline 13 & $\mathrm{~EB}$ & 4 & 338 & Sellado EB \\
\hline 14 & BA & 2,5 & 528 & Sellado BA \\
\hline 15 & $E B$ & 2,5 & 1540 & Ligadura \\
\hline 16 & BA & 2,5 & 453 & Sellado BA \\
\hline 17 & EB & 2 & 1653 & Ligadura \\
\hline 18 & BA & 2,5 & 1353 & Sellado BA \\
\hline 19 & EB & 3 & 528 & Sellado EB \\
\hline 20 & BA & 2 & 275 & Sellado BA \\
\hline 21 & $E B$ & 2,5 & 340 & Sellado EB \\
\hline 22 & BA & 2 & 340 & Sellado BA \\
\hline 23 & $\mathrm{~EB}$ & 3 & 490 & Ligadura \\
\hline 24 & BA & 2 & 528 & Sellado BA \\
\hline 25 & $E B$ & 3 & 378 & Sellado EB \\
\hline
\end{tabular}

EB: electrocoagulación bipolar contralada por temperatura; BA: bisturí armónico.

Tabla II. Resultados del sellado de colaterales de vena safena interna con diferentes dispositivos de hemostasia por energía

\begin{tabular}{|l|c|c|c|}
\hline & $\mathrm{EB}(\mathrm{n}=13)$ & $\mathrm{BA}(\mathrm{n}=12)$ & valor $\mathrm{p}$ \\
\hline Diámetro medio de colateral $(\mathrm{mm})$ & $2,4 \pm 0,7$ & $2,4 \pm 0,6$ & 0,852 \\
\hline Presión media de estallido $(\mathrm{mmHg})$ & $788,9 \pm 455$ & $602,5 \pm 363,1$ & 0,268 \\
\hline Punto de fuga por el dispositivo & $8 / 13(61,5 \%)$ & $12 / 12(100 \%)$ & 0,039 \\
\hline
\end{tabular}

EB: electrocoagulación bipolar contralada por temperatura; BA: bisturí armónico. 

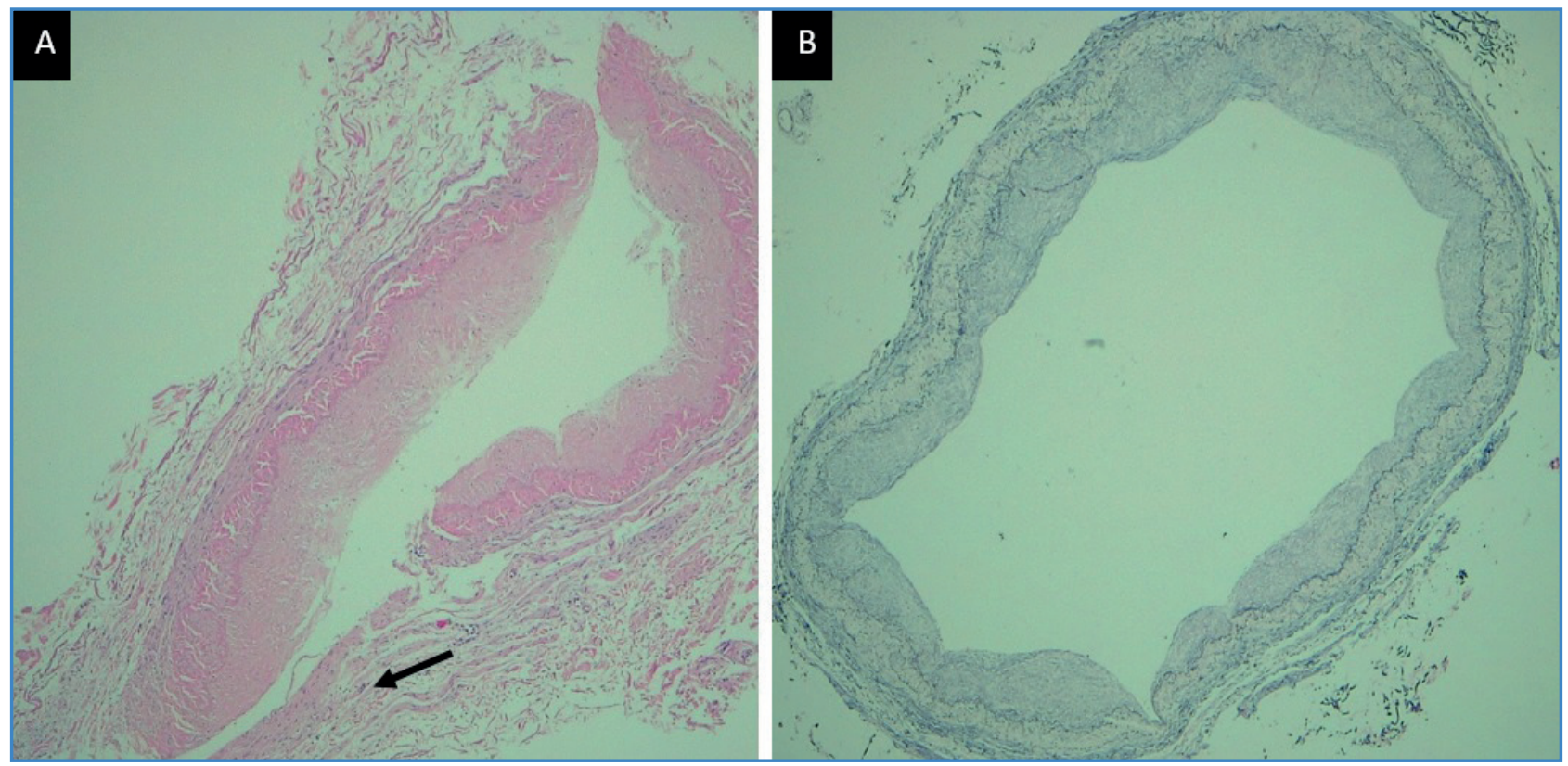

Figura 3. Cortes histológicos de las zonas de sellado. A. Sellado de colateral venosa por EB con tinción hematoxilina-eosina y aumento × 2,5. Flecha negra: zona de sellado. B. Vena safena tras retirada de ligadura con tinción para fibras elásticas y aumento $\times 2,5$.

En los 5 fragmentos en los que no se produjo el estallido en la zona de sellado, sino en la de ligadura, la presión media de rotura fue de 1171,6 \pm $466,8 \mathrm{mmHg}$, significativamente superior al EB y BA $(699 \pm 415,9 \mathrm{mmHg})(p=0,012)$.

En el estudio histológico se observó una necrosis y una desestructuración de las fibras de colágeno y elastina de la pared vascular y la oclusión de la luz del vaso tras el sellado. No se detectaron diferencias morfológicas entre ambos dispositivos, pero sí con respecto a la ligadura convencional, en la que se observó un replegamiento de las capas internas, secundaria a la compresión extrínseca (Fig. 3).

\section{DISCUSIÓN}

Los sistemas de sellado por energía y su eficacia en la hemostasia tisular han sido estudiados tanto en modelos in vitro como en animales y humanos (1-9,13). Los dispositivos EB y BA han demostrado ser una alternativa segura a la ligadura convencional en distintos procedimientos quirúrgicos y presentan otros beneficios frente a las técnicas clásicas, como la disminución del tiempo intraoperatorio $(3,14)$
- la reducción del sangrado intraoperatorio en cirugía laparoscópica (3,15-18).

En la mayoría de estudios comparativos entre ambos dispositivos, la EB presentó un mayor porcentaje de sellado sin fugas y se alcanzaron presiones de estallido superiores a los alcanzados por el BA $(1,3,20,21)$.

Sin embargo, uno de sus inconvenientes es la hemostasia de vasos de mayor tamaño (3,18-21). Así, en la serie de Hruby y cols. (21) se observó un porcentaje de fallos del $85 \%$ en el sellado de arterias de diámetro superior a $7 \mathrm{~mm}$, por lo que se desaconseja su utilización por encima de este umbral.

La principal ventaja del BA frente a la EB es una menor dispersión de la energía a los tejidos circundantes al basarse en un mecanismo de alta vibración. Sin embargo, los resultados de algunos estudios son dispares y no sugieren diferencias clínicas relevantes en la cirugía endoscópica (22,23). Aunque esta tecnología pudiera tener interés en aquellas intervenciones en las que debe realizarse una disección más meticulosa para respetar estructuras nerviosas, el extremo de la pinza que genera el sellado permanece caliente el doble de tiempo que la EB y puede producir lesiones inadvertidas (23). Otro inconveniente, ya comen- 
tado previamente, es una presión de estallido inferior a otros sistemas de energía como es la EB. Sin embargo, esta diferencia solo se hace significativa a partir de diámetros superiores a $3 \mathrm{~mm}$, como se observó en el estudio de Harold y cols. (19).

La mayor parte de estos estudios se basan en modelos arteriales (1,3,4,17-19), aunque existen otros diseños, como el de Landman y cols. (20), en el que se evaluó la hemostasia sobre vasos venosos, y cuyos resultados en cuanto a presión de estallido fueron inferiores, con presiones medias de estallido de 233 mmHg. No obstante, esto puede deberse no solo al mayor diámetro de los vasos utilizados, sino también a la menor resistencia de los fragmentos venosos al ser un sistema de bajas presiones. Esto se refleja también en el estudio de Hruby y cols. (21), en el que se observaron presiones de estallido significativamente inferiores al comparar segmentos arteriales y venosos con el mismo diámetro.

La implantación de estos dispositivos de sellado en el ámbito de la cirugía vascular es inferior a la de otras especialidades con mayor predominio endoscópico, por lo que existen pocos estudios en los que se evalúe su impacto y, en su mayor parte, se centran en valorar su eficacia en la disminución de complicaciones locales en los abordajes quirúrgicos inguinales, como demuestra el de Gouaillier-Vulcain y cols. (24). En este estudio no se encontraron diferencias significativas en el tiempo de cicatrización tras disección femoral con EB, pero sí una menor incidencia de seromas gracias al sellado de los vasos linfáticos con respecto a técnicas clásicas de electrocoagulación.

En nuestro estudio, hemos evaluado la eficacia de los dispositivos de sellado en los fragmentos de VS que se utilizan como injerto para bypass y que, por tanto, se comportarán como una arteria en cuanto a resistencia a altas presiones. Los resultados obtenidos han demostrado que los dispositivos de sellado por energía son eficaces y que permiten soportar presiones de al menos 3 veces la fisiológica, en el caso de la EB, y de aproximadamente 2,5 veces, en el caso del BA.

Las presiones máximas de estallido obtenidas han sido superiores a otros estudios similares, como el de Hruby y cols., con una presión media de estallido de los vasos venosos de $386 \mathrm{mmHg}$ para EB y de $215 \mathrm{mmHg}$ y $160 \mathrm{mmHg}$ para los dos dispositivos de BA; o el de Landman y cols., con presiones de 207 y $342 \mathrm{mmHg}$, respectivamente. Esta mayor presión de estallido probablemente se deba al menor diámetro medio de los vasos analizados en nuestro diseño (igual o inferior a 4 $\mathrm{mm}$ ) en comparación al de los estudios mencionados (6 $\mathrm{mm}$ y 10,9 mm, respectivamente).

También tenemos que destacar, con respecto a otras publicaciones, que en nuestro estudio hemos incluido la ligadura convencional como control interno en paralelo de cada dispositivo. Sin embargo, esto puede suponer una limitación, ya que no puede conocerse exactamente la presión de estallido del dispositivo en el caso de que el fragmento venoso se rompa primero por la zona de sellado de la ligadura, como ha ocurrido en 5 de 13 fragmentos para la EB. Por lo tanto, la presión de estallido que hemos obtenido para la EB probablemente esté infraestimada. No obstante, estas diferencias, así como las observadas con respecto al BA, probablemente no tengan repercusiones clínicas, ya que todas las roturas se produjeron a presiones suprafisiológicas.

Desde el punto de vista histológico, se observó una necrosis y una desestructuración de las capas de la pared vascular y la oclusión de la luz del vaso tras el sellado, similar al observado en otros estudios $(2,25)$. Al comparar las zonas de sellado por energía y la zona de la ligadura, en este caso se observó un replegamiento de las capas internas del vaso sanguíneo, secundaria a la compresión extrínseca de la sutura. No obstante, este hallazgo no condiciona cambios clínicos, como así lo demuestran las altas presiones de estallido.

Un aspecto a destacar de los dispositivos de sellado por energía es su coste comparado con la ligadura convencional. Aunque no existen estudios de coste-eficiencia en el campo de la cirugía vascular, sí se conocen otros estudios en el ámbito de la cirugía general y abdominal en los que se ha demostrado una mayor reducción del tiempo quirúrgico y de pérdidas sanguíneas que pueden compensar el coste inicial de estos dispositivos (26). No obstante, son necesarios futuros estudios que evalúen la eficiencia de dichos dispositivos en la cirugía de revascularización.

Además de la cirugía de revascularización, otra posible aplicación clínica de estos dispositivos 
podría ser el sellado y sección de colaterales venosas en la cirugía de fístulas arteriovenosas autólogas para hemodiálisis, aunque su eficacia y su seguridad aún deben ser evaluadas en futuros estudios.

Nuestro estudio es uno de los primeros ensayos que evalúan la eficacia en el sellado de colaterales venosas para su empleo como injerto en la cirugía de revascularización. Sin embargo, presenta algunas limitaciones en su diseño, como la asignación secuencial consecutiva no aleatorizada. La razón de esta decisión, por motivos de infraestructura y disponibilidad de muestras y dispositivos, es poco probable que haya tenido repercusión en los resultados y su interpretación clínica, ya que en todos los casos se consiguió el sellado a presiones suprafisiológicas.

\section{CONCLUSIONES}

Los resultados de este estudio sugieren que los dispositivos de hemostasia por energía presentan una eficacia y una seguridad no inferiores a las de la ligadura convencional en el sellado de colaterales de vena safena, y que pueden ser útiles, dada su rapidez y fácil manejo, en la preparación del injerto venoso durante la cirugía de revascularización. Aunque el EB mostró una mayor resistencia a la rotura, al producirse el estallido a presiones suprafisiológicas, este hecho podría no tener relevancia clínica.

\section{BIBLIOGRAFÍA}

1. Rajbabu K, Barber NJ, Choi W, et al. To knot or not to knot? Sutureless haemostasis compared to the surgeon's knot. Ann R Coll Surg Engl 2007;89(4):359-62.

2. Lacin T, Batirel HF, Ozer K, et al. Safety of a thermal vessel sealer on main pulmonary vessels. Eur J Cardiothorac Surg 2007;31(3):482-5.

3. Toishi M, Yoshida K, Agatsuma H, et al. Usefulness of vessel-sealing devices for $\leq 7 \mathrm{~mm}$ diameter vessels: a randomized controlled trial for human thoracoscopic lobectomy in primary lung cancer. Interact Cardiovasc Thorac Surg 2014;19(3):448-55.

4. Clements $\mathrm{RH}$, Palepu R. In vivo comparison of the coagulation capability of SonoSurg and Harmonic Ace on $4 \mathrm{~mm}$ and 5 mm arteries. Surg Endosc 2007;21(12):2203-6.

5. Tsunezuka Y, Waseda RI, Yachi T. Electrothermal bipolar vessel sealing device ligaSureVTM for pulmonary ar- tery ligation - burst pressure and clinical experiences in complete videoassisted thoracoscopic major lung resection for lung cancer. Interact Cardiovasc Thorac Surg 2010;11(3):229-33.

6. Katsuno G, Nagakari K, Fukunaga M. Comparison of two different energy-based vascular sealing systems for the hemostasis of various types of arteries: a porcine model-evaluation of LigaSure ForceTriad. J Laparoendosc Adv Surg Tech A 2010;20(9):747-51.

7. Elhao M, Abdallah K, Serag I, et al. Efficacy of using electrosurgical bipolar vessel sealing during vaginal hysterectomy in patients with different degrees of operative difficulty: a randomised controlled trial. Eur J Obstet Gynecol Reprod Biol 2009;147(1):86-90.

8. Lesser TG, Wolfram F, Boltze C. Sealing of pulmonary arteries with LigaSure: in vivo and ex vivo examinations. J Thorac Cardiovasc Surg 2013;145(6):1525-8.

9. Martin ST, Heeney A, Pierce C, et al. Use of an electrothermal bipolar sealing device in ligation of major mesenteric vessels during laparoscopic colorectal resection. Tech Coloproctol 2011;15(3):285-9.

10. Perrault LP, Kollpainter R, Pagé $P$, et al. Techniques, complications, and pitfalls of endoscopic saphenectomy for coronary artery bypass grafting surgery. J Card Surg 2005;20(4):393-402.

11. Allen KB, Heimansohn DA, Robison RJ, et al. Risk factors for leg wound complications following endoscopic versus traditional saphenous vein harvesting. Heart Surg Forum 2000;3(4):325-30.

12. Carpino PA, Khabbaz KR, Bojar RM, et al. Clinical benefits of endoscopic vein harvesting in patients with risk factors for saphenectomy wound infections undergoing coronary artery bypass grafting. J Thorac Cardiovasc Surg 2000;119(1):69-75.

13. Wallwiener CW, Junginger SH, Zubke W, et al. Bipolar vessel sealing: instrument contamination and wear have little effect on seal quality and success in a porcine in vitro model. Langenbecks Arch Surg 2014;399(7):863-71.

14. Ding Z, Wable M, Rane A. Use of Ligasure bipolar diathermy system in vaginal hysterectomy. J Obstet Gynaecol 2005;25(1):49-51.

15. Leonardo C, Guaglianone S, De Carli P, et al. Laparoscopic nephrectomy using Ligasure system: preliminary experience. J Endourol 2005;19(8):976-8.

16. Demirturk F, Aytan H, Caliskan AC. Comparison of the use of electrothermal bipolar vessel sealer with harmonic scalpel in total laparoscopic hysterectomy. J Obstet Gynaecol Res 2007;33:341-5.

17. Lamberton GR, Hsi RS, Jin DH, et al. Prospective comparison of four laparoscopic vessel ligation devices. J Endourol 2008;22(10):2307-12.

18. Richter S, Kollmar O, Schilling MK, et al. Efficacy and quality of vessel sealing: comparison of a reusable with a disposable device and effects of clamp surface geometry and structure. Surg Endosc 2006;20(6):890-4. 
19. Harold KL, Pollinger $\mathrm{H}$, Matthews BD, et al. Comparision of ultrasonic energy, bipolar termal energy, and vascular clips for the hemostasis of small-, medium-, and large-sized arteries. Surg Endosc 2003;17:1228-30.

20. Landman J, Kerbl K, Rehman J, et al. Evaluation of a vessel sealing system, bipolar electrosurgery, harmonic scalpel, titanium clips, endoscopic gastrointestinal anastomosis vascular staples and sutures for arterial and venous ligation in a porcine model. J Urol 2003;169(2):697-700.

21. Hruby GW, Marruffo FC, Durak E, et al. Evaluation of surgical energy devices for vessel sealing and peripheral energy spread in a porcine model. J Urol 2007;178(6): 2689-93.

22. Phillips CK, Hruby GW, Durak E, et al. Tissue response to surgical energy devices. Urology 2008;71(4):744-8.
23. Kim FJ, Chammas MF Jr, Gewehr E, et al. Temperature safety profile of laparoscopic devices: Harmonic ACE (ACE), Ligasure $V(L V)$, and plasma trisector (PT). Surg EndosC 2008;22(6):1464-9.

24. Gouaillier-Vulcain F, Marchand E, Martínez R, et al. Utility of electrofusion for the femoral approach in vascular surgery: a randomized prospective study. Ann Vasc Surg 2015;29(4):801-9.

25. Lesser TG, Wolfram F, Boltze C. Sealing of pulmonary arteries with LigaSure: in vivo and ex vivo examinations. J Thorac Cardiovasc Surg 2013;145(6):1525-8.

26. Pons Y, Gauthier J, Ukkola-Pons E, et al. Comparison of Ligasure Vessel Sealing System, Harmonic Scalpel, and Conventional Hemostasis in Total Thyroidectomy. Otolaryng Head Neck 2009;141(4):496-501. 